\title{
Associação entre via de parto e complicações maternas em hospital público da Grande São Paulo, Brasil
}

\author{
Association between mode of delivery and \\ maternal complications in a public hospital in \\ Greater Metropolitan São Paulo, Brazil
}

\author{
Luís Carlos Machado Junior 1 \\ Christian Eric Sevrin 1 \\ Emerson de Oliveira 1 \\ Heráclito Barbosa de Carvalho 2 \\ Jorge Washington Zamboni 1 \\ José Carlos de Araújo 1 \\ Marilande Marcolin 1 \\ Paulo Caruso ${ }^{1}$ \\ Pedro Ferreira Awada ${ }^{1}$ \\ Ricardo Zanetti Giunta 1 \\ Wirley Munhoz 1 \\ Mauro Sancovski 1 \\ Sérgio Peixoto ${ }^{1}$
}

\section{Introdução}

${ }_{1}^{1}$ Faculdade de Medicina do ABC, Santo André, Brasil.

2 Faculdade de Medicina, Universidade de São Paulo, São Paulo, Brasil.

Correspondência L. C. Machado Junior Faculdade de Medicina do $A B C$.

Rua Alexandre Benois 180 apto. 101, São Paulo, SP 05729-090, Brasil. machado-maria@uol.com.br

\section{Abstract}

The purpose of this study was to assess the relationship between mode of delivery and maternal complications, based on a retrospective cohort of all births at a public hospital in 2003. Complications included: infection, hemorrhage, hysterectomy, uterine rupture, lesions in adjacent organs, deep venous thrombosis, and pulmonary embolism. The analysis used odds ratio (OR), chisquared test, and Fisher's exact test, besides logistic regression. Fifteen complications were identified. Taking vaginal delivery as the reference, an association was found between cesarean section and overall complications. Analysis of confounding showed an association between hypertension, HIV, placenta previa, and abruptio placentae. After controlling for these variables, an association remained between overall complications and cesarean section $(O R=9.7 ; p=0.04)$. Another analysis comparing elective cesareans and vaginal deliveries also showed an increased risk for cesarean $(O R=4.7 ; p=0.02)$. Finally, comparing elective cesareans with trial of labor, we found an increased proportion of complications in elective cesareans, with borderline significance $(O R=3$; $p=0.058$ ). We concluded that cesarean section is associated with maternal morbidity, even after controlling for confounders.

Parturition; Postoperative Complications; Puerperal Disorders
Têm sido freqüentes os debates, em nosso meio e na literatura médica nacional e internacional, sobre os constantes aumentos das taxas de cesárea 1,2,3,4,5,6 bem como, paralelamente, sobre a possibilidade de ampliação das indicações médicas deste procedimento $7,8 \mathrm{e}$ ainda, inclusive, sobre $\mathrm{o}$ direito de a mulher escolher o tipo de parto que deseja 9,10,11,12. Tem também sido documentada, no Brasil, uma maior freqüência de cesáreas nas classes sócio-econômicas mais altas 13,14,15. Um elemento fundamental para esta discussão é o risco de morte e complicações, tanto maternas como neonatais, associado à cesárea, em comparação àquele associado ao parto vaginal.

Grande parte dos estudos abordando este tema tem hoje sua validade questionada por terem avaliado casuísticas de décadas atrás, e muito provavelmente houve, de uma maneira geral uma diminuição dos riscos associados tanto ao parto pela via vaginal quanto à cesárea durante este período.

Nos últimos anos, porém, têm sido retomados os estudos com tais objetivos. Em relação aos riscos neonatais segundo a via de parto existem evidências de maior risco de complicações respiratórias na cesárea eletiva (isto é, aquela realizada fora do trabalho de parto), mesmo nas gestações a termo 16,17. Existem também estudos mostrando maior incidência de recém-nascidos de baixo peso neste tipo de 
cesárea 18,19. Existem evidências sobre maior risco de morte e complicações na via vaginal na apresentação pélvica 20,21. Existem dúvidas sobre a via de parto que oferece menor risco para o prematuro extremo, bem como na gestação a termo na apresentação cefálica 14,22,23,24.

Em relação à mortalidade materna, há o estudo de Lydon-Rochelle et al. 25, feito nos Estados Unidos com 256.471 primíparas, que não encontrou associação significativa entre via de parto e mortalidade materna após ajuste para pré-eclampsia grave e idade materna. A própria autora, porém, faz no texto a ressalva de que, ao desenhar o estudo, tinha a expectativa de um número muito maior de mortes maternas, e de que o pequeno número de mortes resultou então em um baixo poder para a detecção desta associação. Afirma que suas conclusões devem ser interpretadas com cautela. Estudos posteriores mostram resultados diferentes. Harper et al. ${ }^{26}$, nos Estados Unidos, mostraram risco maior de morte associado à cesárea, mesmo após ajuste para doenças maternas, idade materna e idade gestacional, com odds ratio (OR) de 3,9; intervalo de $95 \%$ de confiança (IC95\%) de 2,5-6,1. Deneux-Thoraux et al. 27, na França, estudaram apenas partos em mulheres que não tinham doenças pré-existentes ou complicações obstétricas que aumentam o risco materno. Encontrou risco maior de morte na cesárea $(\mathrm{OR}=3,6$; IC95\%: 2,1-6,2), após ajuste para idade materna, idade gestacional e nacionalidade. O risco aumentado se verificou também ao se analisar apenas as cesáreas eletivas em comparação aos partos vaginais $(\mathrm{OR}=3,1)$.

No Brasil, Kilsztajn et al. 15 estudaram os nascimentos em instituições ligadas ao Sistema Único de Saúde (SUS) no Estado de São Paulo nos anos de 2001, 2002 e 2003, com cerca de um milhão e 100 mil partos, e encontrou também maior risco de morte materna associada à cesárea em relação ao parto vaginal, mesmo após ajuste para doenças e idade materna $(\mathrm{OR}=3,3$; IC95\%: 2,6-4,3).

Em relação à morbidade materna, existem também estudos recentes. Lydon-Rochelle et al. ${ }^{28}$, nos Estados Unidos, estudaram o risco de reinternação após o parto, segundo a via de parto, excluindo as mulheres com doenças pré-existentes. Após ajuste para idade materna, encontrou risco maior de reinternação (inclusive por apendicite e colecistite aguda) associado à cesárea comparada ao parto vaginal espontâneo, com risco relativo (RR) de 1,8 (IC95\%: 1,6-1,9). Allen et al. 29, no Canadá, estudando primíparas, comparou a morbidade da cesárea eletiva com a da "tentativa de parto vaginal", independentemente do tipo de parto ocorrido ("planned vaginal delivery", "trial of labor"). Encontrou, no grupo das cesáreas eletivas, maior risco de morbidade febril puerperal $(\mathrm{RR}=2,2$; IC95\%: 1,1-4,5). Analisando, contudo, o conjunto de todas as complicações estudadas, não encontrou diferença significativa entre e cesárea eletiva e a tentativa de parto vaginal. Burrows et al. ${ }^{30}$, nos Estados Unidos, encontraram maior risco de endometrite, pneumonia e transfusão de sangue, tanto na cesárea de emergência como na eletiva em comparação com o parto vaginal espontâneo. Estes autores, no entanto, não compararam a cesárea eletiva com a tentativa de parto vaginal. Villar et al. 31 , em estudo multicêntrico de tipo ecológico feito em vários países da América Latina, encontraram associação entre cesárea e morte materna e morbidade materna grave, após ajuste para variáveis confundidoras, tanto na cesárea eletiva como na de emergência.

No Brasil, o estudo de Nomura et al. 32 feito em hospital universitário na cidade de São Paulo, não mostrou associação entre via de parto e complicações maternas.

Os estudos de morbidade materna citados acima estudaram as complicações mais imediatamente relacionadas ao parto, ocorridas durante a internação hospitalar ou no período puerperal (o estudo de Lydon-Rochelle et al. 28 incluiu as reinternações até sessenta dias pós-parto). Outros autores estudaram as repercussões de longo prazo relacionadas ao tipo de parto. Descreve-se maior incidência de perda urinária de esforço 33 e incontinência fecal 34 associadas ao parto vaginal, especialmente com o vaginal operatório, e maior risco de placenta prévia, descolamento prematuro de placenta 35 e rotura uterina $36 \mathrm{em}$ gestações subseqüentes associados à cesárea. Thompson et al. 37 organizaram uma coorte que acompanhou mulheres 8, 16 e 24 semanas após o parto e avaliou, por meio de questionário respondido pelas mulheres, a freqüência de sintomas subjetivos e reinternação. Encontrou associação entre cesárea e sintomas de fadiga, falta de sono, problemas intestinais e reinternação, em comparação com o parto vaginal espontâneo. O parto vaginal mostrou associação com dor perineal e perda urinária de esforço, mas no vaginal operatório a associação foi maior. Após seis meses de seguimento, entretanto, a associação com perda urinária de esforço deixou de ser significativa.

Diante do exposto, foi elaborado o presente estudo com o objetivo de comparar o risco de complicações maternas no seu conjunto em partos realizados por via abdominal com aqueles realizados por via vaginal, ocorridas até o momento da alta hospitalar, realizando controles para idade e outros fatores de risco maternos. Também se comparou o risco de complicações da cesárea eletiva aos partos vaginais e da cesárea 
eletiva com a "tentativa de parto vaginal". Não se pretendeu estudar a mortalidade materna, pois o tamanho da amostra não é suficiente para conclusões sobre este evento.

\section{Métodos}

Estudou-se uma coorte retrospectiva formada pelos partos de fetos com $500 \mathrm{~g}$ ou mais, nascidos vivos, durante todo o ano de 2003, no Centro Hospitalar de Santo André, excluindo-se as gestações múltiplas e as internações após partos ocorridos fora do ambiente hospitalar. Esta instituição é um hospital público, mantido pelo município desta cidade, que faz parte do SUS. A equipe de Ginecologia e Obstetrícia faz parte da Faculdade de Medicina do ABC. A equipe é composta por membros do corpo docente, médicos contratados, alunos do quinto e sexto anos, além de residentes de primeiro e segundo anos de Ginecologia e Obstetrícia da faculdade. A equipe de plantão conta com pelo menos dois plantonistas que são especialistas em Ginecologia e Obstetrícia, um residente de primeiro e outro de segundo ano da especialidade, além de alunos do quinto e sexto anos. Nesta especialidade, portanto, a instituição funciona como hospital-escola. O hospital recebe gestantes de baixo risco, mas também é instituição de referência para gestações de alto risco. Logo, atende a uma demanda mista quanto a este aspecto. O levantamento foi feito mediante a revisão manual dos prontuários. A instituição não conta com prontuário informatizado. Foi aplicado aos prontuários um questionário previamente elaborado, abrangendo vários dados maternos e neonatais. O levantamento foi feito apenas pelos autores, não tendo se utilizado do trabalho de alunos, residentes ou de auxiliares contratados.

Em relação à escolha das complicações a serem estudadas, levou-se em conta a importância delas. Foram escolhidas: infecção, hemorragia, trombose venosa profunda, embolia pulmonar, histerectomia, lesão de órgão contíguo e rotura uterina. Em relação aos critérios para defini-las, houve a preocupação de que fossem de tal modo, que contornassem falhas de anotação no prontuário ou aspectos dúbios no diagnóstico. Definiuse, por exemplo, infecção como prolongamento da internação ou reintervenção devido a este diagnóstico. Considerou-se que critérios como elevação de temperatura, hiperemia ou exsudato de ferida operatória ou uso de antibióticos como pouco precisos para a definição deste diagnóstico. Em relação à hemorragia, escolheu-se transfusão sanguínea durante a internação para o parto como critério, exceto se indicada por anemia previamente existente. Em relação às outras complicações, histerectomia (por qualquer indicação), trombose venosa profunda, embolia pulmonar, lesão de órgão contíguo e rotura uterina, considerou-se que são bem definidas o suficiente e de tal importância para que o critério fosse apenas a presença do registro delas no prontuário.

Foram também levantadas algumas características maternas que poderiam estar associadas a complicações e também estar associadas às indicações de cesáreas, podendo assim funcionar como variáveis confundidoras, com o objetivo de serem feitos controles para estas variáveis. O objetivo desses controles foi chegar o mais próximo possível do risco que pode ser atribuído ao procedimento "operação cesariana" propriamente dito em comparação ao parto vaginal, de modo independente de outros fatores de risco eventualmente associados. As variáveis estudadas são do tipo dicotômicas, isto é, em sua maioria, observa-se a presença ou ausência do item pesquisado. Levantou-se: idade (até 35 anos ou mais de 35), paridade (primíparas $v$ s. o restante das paridades e cinco ou mais partos $v s$. o restante das paridades). Com o mesmo objetivo, levantou-se a ocorrência das seguintes doenças: síndrome hipertensiva (englobando hipertensão arterial crônica, pré-eclampsia isolada ou associada à hipertensão crônica), diabetes mellitus (todos os tipos), anemia, cardiopatia, soropositividade para o vírus da imunodeficiência humana (HIV), além de placenta prévia e descolamento prematuro de placenta.

Também se verificou o caráter de indicação da cesárea: se foi realizada previamente ao trabalho de parto (como, por exemplo, no caso de duas ou mais cesáreas anteriores ou quando se indica a interrupção da gestação e há contra-indicação para indução do parto) ou se foi realizada por intercorrências detectadas durante a evolução do trabalho de parto (desproporção céfalo-pélvica ou alteração de cardiotocografia intraparto, por exemplo). Isto foi feito para se avaliar o risco das cesáreas eletivas, que foram definidas, então, como as realizadas fora do trabalho de parto e na ausência de descolamento prematuro de placenta ou placenta prévia.

Como a cesárea muitas vezes é indicada para contornar problemas que surgem durante a evolução de um parto vaginal, as eventuais complicações decorrentes da cesárea podem ser consideradas como conseqüentes à decisão de tentar um parto vaginal. Em outras palavras, quando se decide por um parto vaginal, nunca há certeza sobre se ele será vaginal ou cesáreo. Conseqüentemente, existe interesse, do ponto de vista de auxiliar nas decisões clínicas nas diversas situações cotidianas, de comparar os riscos de complicações maternas ao se tentar um parto vaginal 
em comparação com o risco de se indicar uma cesárea sem esta tentativa. Propusemo-nos então a comparar a cesárea eletiva como definido acima com as "tentativas de parto vaginal" ("trial of labor", "planned vaginal delivery”), ou seja, com todas as pacientes com as quais se tentou o parto vaginal, independentemente de o parto ter sido possível ou ter sido necessária uma cesárea, já que nosso prontuário permite que se verifique se foi ou não feita tal tentativa.

Na instituição, o tempo proposto para a alta em partos sem complicações é de 48 horas, tanto para cesárea quanto para parto normal ou fórcipe. As complicações descritas acima foram pesquisadas até o momento da alta, uma vez que existe dificuldade em acompanhar as pacientes depois dela.

Foi utilizado o programa Epidata (Epidata Association, Odense, Dinamarca) para a elaboração do banco de dados. Os dados foram digitados e posteriormente ajustados em sua consistência antes do procedimento das análises. Foi utilizado o teste de qui-quadrado de Pearson e teste de Fisher para avaliar diferenças entre variáveis categóricas, estabelecendo-se uma significância estatística (alfa) de 5\%. Utilizou-se como medida de efeito a OR com IC95\%. Para controle de possíveis variáveis de confusão, foi realizada análise multivariada por meio de regressão logística em que a significância estatística foi avaliada pelo teste de razão de verossimilhança (likelihood ratio test), considerando-se como significante $\mathrm{p}<0,05$.

Foram utilizados os pacotes estatísticos Epi Info (Centers for Disease Control and Prevention, Atlanta, Estados Unidos) e Stata 8.0 (Stata Corp., College Station, Estados Unidos) para digitação dos dados e execução das análises descritivas e analíticas.

A digitação foi feita por três dos autores. $\mathrm{O}$ levantamento foi também utilizado para analisar os resultados neonatais, os quais se pretende divulgar em outra publicação, e, por isso, não se pretendeu apresentá-los ou discuti-los no presente estudo. O protocolo do estudo foi aprovado pelo Comitê de Ética em Pesquisa da Faculdade de Medicina do ABC sob número 40/05.

\section{Resultados}

Foram estudados 1.530 nascimentos consecutivos de janeiro a dezembro de 2003. A idade média das parturientes foi de 25,4 anos, variando de 13 a 47 anos. Eram primíparas 34,5\% e 4,5\% tinham cinco ou mais partos anteriores. A freqüência de síndrome hipertensiva foi de $11,7 \%$. A freqüência de cesáreas foi de 39,5\%, e de fórcipe de 8,6\%.
Como comparação, a freqüência de cesáreas em parturientes do SUS no Estado de São Paulo no ano de 2003 foi de 32,9\%. Porém, nossa proporção de gestantes de alto risco é provavelmente maior que a da população do estado como um todo. No mesmo ano, a freqüência de cesáreas no estado, incluindo os setores público e privado, foi de $51,4 \%$ e no setor privado exclusivamente de $80,4 \%$. Em nossa série, nas mulheres nas quais se tentou a via vaginal, a proporção de sucessos foi de $88,2 \%$. Entre pacientes com uma cesárea anterior, $38,5 \%$ deram à luz por via vaginal. Nas pacientes com uma cesárea anterior nas quais se tentou a via vaginal, o procedimento foi possível em $74,8 \%$. Não houve morte materna nesta série. Foram encontradas 15 complicações. Duas mulheres tiveram mais de uma complicação, o que resulta numa taxa de $0,85 \%$ do total dos partos. Como não houve diferença significativa entre os partos fórcipe e normal quanto às complicações em geral, englobamos estas duas modalidades como parto vaginal. A Tabela 1 mostra a distribuição das complicações segundo a via de parto. Não houve casos de rotura uterina, trombose venosa profunda, embolia pulmonar ou lesão de órgão contíguo. Analisando-se cada complicação separadamente, houve diferença significativa entre as vias vaginal e abdominal apenas para transfusão sanguínea. Analisadas, contudo, em conjunto, a freqüência no parto cesárea foi de $2 \%$ e na via vaginal de $0,3 \%$, configurando uma associação significativa ( $\mathrm{OR}=6,23 ; \mathrm{p}=0,002$ ). Utilizaram-se então as complicações no seu conjunto para as análises subseqüentes. Analisou-se a seguir a associação do conjunto das complicações com as possíveis variáveis confundidoras descritas anteriormente. Encontrou-se associação com síndrome hipertensiva, soropositividade para HIV, placenta prévia e descolamento prematuro de placenta (Tabela 2). Observa-se uma associação importante entre placenta prévia, descolamento prematuro de placenta e soropositividade para HIV com as complicações. Das 15 complicações encontradas, 6 (40\%) ocorreram em mulheres com estas co-morbidades. Por outro lado, a soma de todas as mulheres que apresentaram tais condições foi de 34, correspondendo a apenas $2,2 \%$ do total de partos. Além disso, nas três condições citadas acima, existe indicação de cesárea na grande maioria das vezes (na instituição, indica-se cesárea às mulheres soropositivas para HIV). Decidiu-se, então, excluir da análise as mulheres que apresentavam estas três condições, para evitar que a associação da cesárea com complicações fosse confundida pela associação da cesárea com estas co-morbidades. Restaram então 1.496 partos. Neste grupo restante, manteve-se a associação entre o conjunto 
Tabela 1

Tipos de complicações por via de parto nas 1.530 parturientes estudadas (926 partos vaginais e 604 cesáreos) e cálculo do risco associado ao tipo de parto.

\begin{tabular}{|c|c|c|c|c|c|c|c|}
\hline \multirow[t]{3}{*}{ Complicações * } & \multicolumn{4}{|c|}{ Tipo de parto } & \multirow[t]{3}{*}{ Valor de $\mathrm{p}$} & \multirow[t]{3}{*}{ OR } & \multirow[t]{3}{*}{ IC95\% } \\
\hline & \multicolumn{2}{|c|}{ Vaginal } & \multicolumn{2}{|c|}{ Cesáreo } & & & \\
\hline & $n$ & $\%$ & $n$ & $\%$ & & & \\
\hline Infecção & 3 & 0,3 & 5 & 0,8 & 0,2 & 2,6 & $0,6-10,8$ \\
\hline Hemorragia ** & 0 & - & 6 & 1,0 & 0,002 & 2,6 & $2,4-2,7$ \\
\hline Histerectomia & 0 & - & 1 & 0,2 & 0,2 & 2,5 & $0,4-14,4$ \\
\hline Todas ** & 3 & 0,3 & 12 & 2,0 & 0,002 & 6,2 & $1,7-34,5$ \\
\hline
\end{tabular}

* Não houve casos de trombose venosa profunda, embolia pulmonar, rotura uterina ou lesão de órgão contíguo;

** Significância estatística $p<0,05$.

Tabela 2

Co-morbidades observadas que se associam a complicações do parto nas 1.530 parturientes estudadas (926 partos vaginais e 604 cesáreos) e cálculo do risco de complicações associado a elas.

\begin{tabular}{|c|c|c|c|c|c|c|}
\hline \multirow[t]{2}{*}{ Variável } & \multirow[t]{2}{*}{$n$ * } & \multicolumn{2}{|c|}{ Complicações } & \multirow[t]{2}{*}{ Valor de $p$} & \multirow[t]{2}{*}{ OR } & \multirow[t]{2}{*}{ IC95\% } \\
\hline & & $\mathbf{n}$ & $\%$ ** & & & \\
\hline Síndromes hipertensivas & 179 & 5 & 2,8 & 0,002 & 3,9 & $1,7-6,8$ \\
\hline Sorologia positiva para HIV & 13 & 2 & 15,4 & 0,006 & 24,8 & $2,4-134,8$ \\
\hline Placenta prévia & 6 & 3 & 50,0 & $<0,0001$ & 126,0 & $17,3-1218,5$ \\
\hline Descolamento prematuro de placenta & 15 & 1 & 6,7 & 0,02 & 7,7 & $1,2-58,7$ \\
\hline
\end{tabular}

Nota: a proporção de complicações no total das parturientes foi de $0,85 \%$.

* Número de parturientes com a co-morbidade;

** Porcentagem de complicações dentro do grupo com a respectiva co-morbidade.

das complicações e cesárea $(\mathrm{OR}=12,8 ; \mathrm{p}=0,002$ IC95\%: 1,6-103,3). Repetiu-se, em tal caso, neste grupo, a pesquisa de associação entre as características maternas e o conjunto das complicações, como realizado anteriormente com o grupo completo. Manteve-se apenas a associação com síndrome hipertensiva $(\mathrm{OR}=6,2 ; \mathrm{p}=0,002$; IC95\%: 1,6-23,5). Realizou-se, nesse momento, a regressão logística incluindo síndrome hipertensiva e via de parto, novamente para se evitar que a associação da cesárea com complicações fosse confundida pela maior freqüência de cesáreas na síndrome hipertensiva. Manteve-se a associação entre cesárea e o conjunto das complicações (OR = 9,7; $\mathrm{p}=0,04 ;$ IC95\%: 1,2-80,4), como pode ser visto na Tabela 3 .

Levando-se em conta o risco supostamente maior da cesárea de emergência em comparação com a cesárea eletiva, decidiu-se comparar o risco das cesáreas eletivas, como definidas em "métodos", com o dos partos vaginais, excetuando-se também deste último grupo os casos de placenta prévia e descolamento prematuro de placenta. Também neste grupo de cesáreas encontrou-se associação com o conjunto das complicações $(\mathrm{OR}=4,7 ; \mathrm{p}=$ 0,02; IC95\%: 1,1-19,9). Repetiu-se neste grupo a análise da associação das possíveis variáveis confundidoras descritas acima com o conjunto das complicações. Não se encontrou associação.

Finalmente, realizou-se comparação entre as cesáreas eletivas com o grupo das "tentativas de parto vaginal”, como foram definidas também na seção "métodos", novamente excluindose do último grupo os casos de placenta prévia e descolamento prematuro de placenta. Encontrou-se maior proporção de complicações no grupo das cesáreas eletivas, porém com significância estatística limítrofe $(\mathrm{OR}=3 ; \mathrm{p}=0,058$; IC95\%: 0,9-9,9).

\section{Discussão}

Este estudo mostra que, em nossa instituição, a cesárea oferece maior risco de complicações comparada ao parto vaginal. Este risco aumentado se manteve significativo após controle para doenças 
Relação entre via de parto e o conjunto das complicações nas três análises realizadas.

\begin{tabular}{lcccc}
\hline Análises realizadas & OR bruta & OR ajustada * & IC95\% & Valor de $\mathbf{p}$ \\
\hline Cesárea vs. parto vaginal & 12,8 & 9,7 & $1,2-80,4$ & 0,040 \\
Cesárea eletiva vs. parto vaginal & 4,7 & - & $1,9-19,9$ & 0,020 \\
Cesárea eletiva vs. tentativa de parto vaginal & 3 & - & $0,9-9,9$ & 0,058 \\
\hline
\end{tabular}

* Ajustado por hipertensão arterial sistêmica.

maternas, idade ou paridade. Também demonstra que o maior risco de complicações não está associado apenas à cesárea de emergência.

Defende-se o conceito de que a cesárea de emergência oferece maior risco de morte e complicações para a mãe que a cesárea eletiva 32 , e muitos estudos confirmam este conceito, mas não todos. No estudo de Deneux-Thoraux et al. 27 , por exemplo, apesar de haver uma proporção maior de mortes no grupo das cesáreas de emergência em comparação com o das eletivas, a diferença não foi significativa. Também em nosso estudo não houve diferença significativa de complicações analisadas no seu conjunto entre as cesáreas eletivas e as de emergência $(p=0,8)$.

Se é de fato verdade que a cesárea de emergência tem maior risco de complicações que a eletiva, coloca-se a seguinte questão: mesmo assumindo-se que a cesárea eletiva tem maior risco que o parto vaginal (o que foi verificado em nosso estudo), como uma parte das "tentativas de parto vaginal” resultarão em cesáreas de emergência, é possível que o conjunto das "tentativas", englobando as bem sucedidas e as que resultaram em cesárea, tenham um risco de complicações e morte igual ou maior que o da cesárea eletiva 7 . A constatação de tal resultado poderia ser usada como argumento a favor da cesárea eletiva, substituindo o parto vaginal 32 . Nosso material, no entanto, não dá suporte à hipótese. Verificouse maior proporção de complicações nas cesáreas eletivas em comparação com as tentativas de parto vaginal. Apesar de o resultado não ter sido significativo, levando-se em conta o fato de estar no limite da significância ( $\mathrm{p}=0,058$ ), e os resultados do conjunto das demais análises realizadas, podemos supor que existe de fato maior risco na cesárea eletiva, e que com uma amostra maior, obter-se-ia um resultado significativo. Em outras palavras, nosso estudo mostra que, pelo menos nesta casuística, existem razões técnicas (diminuição de riscos) para se tentar um parto vaginal quando as condições assim o permitem, embora este seja mais trabalhoso, incerto e demorado, e que esta tentativa não serve apenas para atender a uma eventual "preferência" da parturiente pela via vaginal.

Além do risco relativo de complicações na comparação entre as duas vias de parto, para a tomada de decisões clínicas importa também o risco absoluto relacionado a cada tipo de procedimento. Em nosso material, a incidência das complicações no seu conjunto na cesárea foi de $2 \%$. Mesmo se levando em conta que provavelmente algumas complicações não foram detectadas (por exemplo, as ocorridas após a alta e as que não foram estudadas, como cefaléia pósraquianestesia, hematoma em ferida cirúrgica, pneumonia), podemos considerar uma incidência baixa. Levando-se em conta que a maior parte das complicações não foi grave e que nenhuma resultou em morte, é uma incidência relativamente baixa para se decidir por uma cesárea diante de situações de risco associadas ao parto vaginal, às alterações da evolução deste ou às alterações do bem estar fetal. Diante da solicitação de cesárea por parte da paciente, porém, as questões envolvidas são mais complexas, envolvem o aspecto de valores e transcendem a avaliação objetiva de riscos. O conhecimento dos riscos, entretanto, também é aspecto importante na decisão, tanto do médico quanto da paciente.

Do mesmo modo, pode-se considerar que a incidência das complicações mais importantes, de $0,85 \%$ do total dos partos, foi baixa, ainda que se tenha em conta a provável subestimativa por causa das perdas. Esta baixa incidência foi também constatada e comentada pelos autores de outros estudos 30,32. Tais números enfraquecem o argumento de que o maior risco associado à cesárea, como detectado em nosso estudo, se deveria às condições por vezes inadequadas de atendimento no setor público, pois verificamos este risco aumentado mesmo em um contexto de baixa incidência de complicações.

O estudo de Nomura et al. ${ }^{32}$, com 1.748 nascimentos em 2002, não mostrou diferença significativa entre as complicações das vias abdominal e vaginal. A autora, no entanto, para a análise, dividiu as complicações em dez tipos diferentes de 
evento, fato que fez com que a associação de cada uma delas com a via de parto não fosse significativa. Se, utilizando-se os dados apresentados no seu estudo, fizermos uma análise com o conjunto das complicações, verificaremos que existe associação significativa. De nosso ponto de vista, o risco das complicações no seu conjunto tem mais sentido na tomada de decisões clínicas que o risco de cada uma delas separadamente.

Os pontos frágeis deste estudo são três. Em primeiro lugar, a necessidade de levantamento manual de prontuários, pela não disponibilidade de prontuário informatizado, limitou a quantidade de partos estudados. Todavia, ao estudarmos o conjunto das complicações, conseguimos vários resultados significativos e, assim, foi possível fazer várias análises. Além disto, como o prontuário eletrônico freqüentemente é elaborado com base num prontuário preenchido manualmente, é comum que este último tenha mais dados que o primeiro. Caso estudássemos mais partos, poderíamos conhecer melhor a associação de cada complicação com a via de parto. Em segundo lugar, o fato de as pacientes não serem acompanhadas após a alta fez com que não se detectasse parte das complicações. A maior parte dos estudos discutidos acima que tratam de morbidade, porém, sofrem de limitações semelhantes quanto ao último aspecto. Os estudos de Allen et al. 29 e Burrows et al. 30 acompanharam os casos até a alta. $\mathrm{O}$ estudo de Lydon-Rochelle et al. ${ }^{28}$ analisou apenas as reinternações, não incluindo as complicações que não se enquadraram nesta categoria. O estudo de Nomura et al. 32 incluiu as complicações ocorridas no momento do parto e as que necessitaram de reinternação, mas não informa qual a proporção das pacientes que foi acompanhada e por quanto tempo. Mesmo se nosso estudo fosse prospectivo, uma vez que se estudou a totalidade dos partos de feto único, e não um subgrupo restrito de partos, seria pouco viável acompanhar tamanho número de pacientes (1.530) por um período maior, fora do ambiente hospitalar. Por último, o caráter retrospectivo do estudo nos levou a utilizar, para a definição de infecção e hemorragia, critérios que contornassem eventuais falhas de anotação no prontuário (vide Métodos). A definição dessas complicações deste modo pode ter deixado de detectar casos mais leves de infecção que não exigiram prolongamento da internação, ou casos mais leves de hemorragia que não necessitaram de transfusão de sangue. O caráter retrospectivo também pode ter contribuído para que se perdessem alguns casos menos importantes de lesões de órgãos contíguos, como, por exemplo, uma lesão de esfíncter anal prontamente corrigida. Podemos supor com razoável segurança, no entanto, que nenhuma complicação importante ocorrida até a alta foi perdida. Em relação às complicações como histerectomia, transfusão de sangue, lesão de órgãos como bexiga, ureter, reto ou alça intestinal, trombose venosa profunda ou embolia pulmonar (no caso das duas últimas, se o diagnóstico tiver sido feito), podemos estar seguros de que certamente estariam anotadas no prontuário, desde que tenham ocorrido ou tenham sido constatadas durante a internação na qual ocorreu o parto. Além disso, como o foco principal do estudo é a relação entre os riscos da via abdominal em comparação à vaginal, podemos supor que a perda de alguns casos de complicação não mudou de modo substancial esta relação porque provavelmente foram perdidos casos das duas vias de parto de modo semelhante.

O ponto forte do estudo é o fato de ter sido fundamentado no prontuário médico completo, o que possibilitou o controle para várias possíveis variáveis confundidoras e também a comparação com o grupo da tentativa de parto vaginal, análise que poucos estudos fizeram.

Como foi colocado anteriormente em $M e ́$ todos, não se pretendeu neste estudo analisar nem tampouco discutir a questão dos riscos neonatais associados à via de parto, visto que se trata de tema extenso. Avaliamos que não haveria espaço em um artigo para se discutir conjuntamente a questão materna e neonatal, por mais que ambas as questões estejam bastante relacionadas. Contudo, pretendemos abordar esta questão em outro artigo, estudando esta mesma casuística.

A cesárea é procedimento indispensável na obstetrícia contemporânea, e é freqüentemente utilizada para a resolução de situações de risco tanto para a mãe quanto para o feto. Porém, é necessário o conhecimento profundo de seus riscos e implicações para uma indicação médica adequada e inclusive para que, em caso de solicitação de cesárea por parte da mulher, caso se considere a solicitação aceitável, o médico possa fazer um aconselhamento adequado a fim de que a mulher possa tomar uma decisão mais consciente.

\section{Conclusão}

Encontrou-se maior risco de complicações na cesárea comparada ao parto vaginal, risco este que se manteve após controle para idade, paridade e doenças maternas. Verificou-se também maior risco nas cesáreas eletivas em comparação aos partos vaginais e maior proporção de complicações, no limite da significância estatística, nas cesáreas eletivas em comparação à "tentativa de parto vaginal". 


\section{Resumo}

O estudo objetivou avaliar associação entre via de parto e complicações maternas. Realizou-se coorte retrospectiva com partos ocorridos durante o ano de 2003, em um hospital público. As complicações avaliadas foram: infecção, hemorragia, histerectomia, rotura uterina, lesão de órgão contíguo, trombose venosa profunda e embolia pulmonar. Utilizou-se a odds ratio $(\mathrm{OR})$ e os testes de qui-quadrado de Pearson e de Fisher, além da regressão logística. Estabeleceu-se o nivel de 0,05 como significante. Foram encontradas 15 complicações. Tomando-se o parto vaginal como referência, encontrou-se associação entre cesárea e as complicações tomadas em conjunto. Analisando-se variáveis confundidoras, encontrou-se associação das complicações com hipertensão, soropositividade para HIV, placenta prévia e descolamento prematuro de placenta. Após controle para estas quatro variáveis, manteve-se a associação entre cesárea e complicações $(\mathrm{OR}=9,7 ; p=0,04)$. Encontrou-se também associação entre complicações e cesárea eletiva comparada ao parto vaginal $(O R=4,7 ; p=0,02)$, e maior proporção de complicações, no limite da significância estatística, nas cesáreas eletivas comparadas à "tentativa de parto vaginal" (OR = 3; $p=0,058)$. Conclui-se que a cesárea associa-se a complicações maternas, mesmo após a realização de vários ajustes.

Parto; Complicações Pós-Operatórias; Transtornos Puerperais

\section{Colaboradores}

L. C. Machado Junior participou da idealização, revisão de literatura, desenho, levantamento, digitação, análise estatística, elaboração do texto e coordenação. C. E. Sevrin e J. C. Araújo colaboraram no levantamento, coordenação e revisão da literatura. E. Oliveira contribuiu no levantamento, digitação do artigo e análise estatística. H. B. Carvalho participou do desenho do artigo, na adaptação aos programas estatísticos e análise estatística. J. W. Zamboni contribuiu no levantamento, desenho do artigo e revisão da literatura. M. Marcolin e W. Munhoz contribuíram no levantamento e desenho do artigo. P. Caruso participou do levantamento e da elaboração do texto. P. F. Awada colaborou no levantamento, digitação e revisão da literatura. R. Z. Giunta participou no levantamento e revisão da literatura. M. Sancovski e S. Peixoto participaram da revisão final do artigo.

\section{Agradecimentos}

Aos funcionários do Serviço de Arquivo Médico do Centro Hospitalar de Santo André, especialmente à Sra. Maria Elze da Silva Coutinho, pelo importante apoio, sem o qual este trabalho não seria possível. À Sueli Aparecida Bispo dos Santos, médica neonatologista e diretora do berçário do Centro Hospitalar de Santo André, pelo apoio prestado.

\section{Referências}

1. What is the right number of cesarean sections? Lancet 1997; 349:815.

2. Sachs BP, Castro MA. The risks of lowering the cesarean-delivery rate. N Engl J Med 1999; 340:54-7.

3. Hopkins K. Are Brazilian women really choosing to deliver by cesarean? Soc Sci Med 2000; 51:725-40.

4. Belizán JM, Althabe F, Barros FC, Alexander S. Rates and implications of caesarean section in Latin America: an ecological study. BMJ 1999; 319:1397-400.

5. Althabe F, Belizán JM, Villar J, Alexander S, Bergel E, Ramos S, et al. Mandatory second opinion to reduce rates of unnecessary caesarean sections in Latin America: a cluster randomised controlled trial. Lancet 2004; 363:1934-40.

6. Chaillet N, Dumont A. Evidence-based strategies for reducing cesarean section rates: a meta-analysis. Birth 2007; 34:53-64.
7. Feldman GB, Freiman JA. Prophylatic cesarean section at term? N Engl J Med 1985; 312:1264-7.

8. Matthews TG, Crowley P, Chong A, McKenna P, McGarvey C, O’Regan M. Rising caesarean section rates: a cause for concern? BJOG 2003; 110:346-9.

9. Hall MH. When a woman asks for a caesarean section. Br Med J (Clin Res Ed) 1987; 294:201-2.

10. Paterson-Brown S. Should doctors perform an elective caesarean section on request? Yes, as long as the woman is fully informed. BMJ 1998; 317:462-3.

11. Amu O, Rajendran S, Bolaji II. Should doctors perform an elective caesarean section on request? Maternal choice alone should not determine method of delivery. BMJ 1998; 317:463-5.

12. Conselho Regional de Medicina do Estado de São Paulo. Cesárea a pedido, atender ou não. Ser Médico 2004; 28:24-31. 
13. Fabri RH, Murta EF. Socioeconomic factors and cesarean section rates. Int J Gynaecol Obstet 2002; 76:87-8.

14. Kilsztajn S, Lopes ES, Carmo MSN, Reyes AMA. Vitalidade do recém-nascido por tipo de parto no Estado de São Paulo, Brasil. Cad Saúde Pública 2007; 23:1886-92.

15. Kilsztajn S, Carmo MS, Machado LC, Lopes ES Lima LZ. Caesarean sections and maternal mortality in São Paulo. Eur J Obstet Gynecol Reprod Biol 2007; 132:64-9.

16. Madar J, Richmond S, Hey E. Surfactant-deficient respiratory distress after elective delivery at "term". Acta Paediatr 1999; 88:1244-8.

17. Zanardo V, Simbi AK, Franzoi M, Solda G, Salvadori A, Trevisanuto D. Neonatal respiratoy morbidity risk and mode of delivery at term: influence of timing of elective caesarean delivery. Acta Paediatr 2004; 93:643-7.

18. Murta EFC, Freire GC, Fabri DC, Fabri RH. Could elctive cesarean sections influence the birth weight of full-term infants? São Paulo Med J 2006; 124:313-5.

19. Barbieri MA, Silva AAM, Bettiol H, Gomes UA. Risk factors for the increasing trend in low birth weight among live births born by vaginal delivery, Brazil. Rev Saúde Pública 2000; 34:596-602.

20. Hannah ME, Hannah WJ, Hewson SA, Hodnett ED, Saigal S, Willan AR. Planned caesarean section versus vaginal birth for breech presentation at term: a randomised multicentre trial. Term Breech Trial Collaborative Group. Lancet 2000; 356:1375-83.

21. Hofmeyr GJ, Hannah ME. Planned caesarean section for term breech delivery (Cochrane Review) In: The Cochrane Library, Issue 4, 2007. Oxford: Update Software.

22. Grant A, Glazener CMA. Elective caesarean versus expectant management for delivery of the small baby (Cochrane Review) In: The Cochrane Library, Issue 2, 2008. Oxford: Update Software.

23. Machado CJ, Hill K. Maternal, neonatal and community factors influencing neonatal mortality in Brazil. J Biosoc Sci 2005; 37:193-208.

24. Bobadilha H, Walker CJA. Early neonatal mortality and cesarean delivery in Mexico City. Am J Obstet Gynecol 1991; 164:22-8.

25. Lydon-Rochelle M, Holt VL, Easterling TR, Martin DP. Cesarean delivery and postpartum mortality among primiparas in Washington State, 1987-1996 (1). Obstet Gynecol 2001; 97:169-74.
26. Harper MA, Byington RP, Espeland MA Naughton M, Meyer R, Lane K. Pregnancy-related death and health care services. Obstet Gynecol 2003; 102:273-8.

27. Deneux-Thoraux C, Carmona E, Bouvier-Colle M, Bréart G. Postpartum maternal mortality and cesarean delivery. Obstet Gynecol 2006; 108:541-8.

28. Lydon-Rochelle M, Holt VL, Martin DP, Easterling T. association between method of delivery and maternal rehospitalization. JAMA 2000; 283:2411-6.

29. Allen VM, O'Connell CM, Liston RM, Baskett TF Maternal morbidity associated with cesarean delivery without labor compared with sponteneous onset of labor at term. Obstet Gynecol 2003; 102:477-82.

30. Burrows LJ, Meyn LA, Weber AM. Maternal morbidity associated with vaginal versus cesarean delivery. Obstet Gynecol 2004; 103(5 Pt 1):907-12.

31. Villar J, Valladares E, Wojdyla D, Zavaleta N, Carroli G, Velazco A, et al. Ceasarean delivery rates and pregnancy outcomes: the 2005 WHO global survey on maternal and perinatal health in Latin America. Lancet 2006; 367:1819-29.

32. Nomura RMY, Alves EA, Zugaib M. complicações maternas associadas ao tipo de parto em hospital universitário. Rev Saúde Pública 2004; 38:9-15.

33. Foldspang A, Mommsen S, Djurhuus JC. Prevalent urinary incontinence as a correlate of pregnancy, vaginal childbirth, and obstetric techniques. Am J Public Health 1999; 89:209-12.

34. Zetterström JP, López A, Anzén B, Dolk A, Norman M, Mellgren A. Anal incontinence after vaginal delivery: a prospective study in primiparous women. Br J Obstet Gynaecol 1999; 106:324-30.

35. Lydon-Rochelle M, Holt VL, Easterling TR, Martin DP. First-birth cesarean and placental abruption or previa at second birth (1). Obstet Gynecol 2001; 97(5 Pt 1):765-9.

36. Lydon-Rochelle M, Holt VL, Easterling TR, Martin DP. Risk of uterine rupture during labor among women with a prior cesarean delivery. N Engl J Med 2001; 345:3-8.

37. Thompson JF, Roberts CL, Currie M, Ellwood DA. Prevalence and persistence of health problems after childbirth: association with parity and method of birth. Birth 2002; 29:83-94.

Recebido em 28/Nov/2007

Versão final reapresentada em 04/Jun/2008

Aprovado em 10/Jun/2008 